\title{
Tsafon
}

Revue d'études juives du Nord

$79 \mid 2020$

Les Juifs du Nord et du Pas-de-Calais dans la Shoah

\section{Une famille du Nord et du Pas-de-Calais dans la} Shoah

\author{
La famille Sokolski
}

David Laboux

\section{OpenEdition}

\section{Journals}

Édition électronique

URL : https://journals.openedition.org/tsafon/2921

DOI : $10.4000 /$ tsafon.2921

ISSN : 2609-6420

\section{Éditeur}

Association Jean-Marie Delmaire

Édition imprimée

Date de publication : 1 juillet 2020

Pagination : 153-161

ISSN : 1149-6630

\section{Référence électronique}

David Laboux, «Une famille du Nord et du Pas-de-Calais dans la Shoah », Tsafon [En ligne], 79 | 2020, mis en ligne le 01 juillet 2020, consulté le 24 juin 2021. URL : http://journals.openedition.org/tsafon/ 2921 ; DOI : https://doi.org/10.4000/tsafon.2921 


\title{
Une famille du Nord et du Pas-de-Calais dans la Shoah
}

\author{
La famille Sokolski
}

David Laboux*

\section{Une famille juive de Douai avant la guerre}

Adolphe Sokolski né le 17 janvier 1898 à Varsovie, est représentatif de ces nombreux Juifs polonais émigrés dans le Nord et le Pas-de-Calais, fuyant l'antisémitisme féroce de la Pologne des années trente, et considérant la République française tel un pays de rêve et de liberté. Dénommé Abraham, dans les années vingt, il prend le prénom d'Adolphe «plus français » selon son fils Claude Sokolski ${ }^{1}$. Au début des années trente (année inconnue par son fils Claude), Adolphe se marie dans le Nord avec Sosia Gruszka, née le 17 mai 1918, à Siedlice en Pologne. Le couple vit modestement : Sosia est mère au foyer et Adolphe est tailleur.

Le couple réside au 4 rue du Champ-Fleuri à Douai. Rapidement, la famille s'agrandit. Trois enfants naissent à Lille et Douai et sont ainsi Français : Jacqueline en 1936 à Lille, Arlette en 1938 et Francine en 1939, à Douai. «C'est mon père qui les habillait» précise Claude Sokolski ${ }^{2}$.

\footnotetext{
* Musée de l'Holocauste et des Droits de l'homme de Malines, Belgique. L'article paraîtra aussi dans le bulletin de la Fondation Auschwitz, Belgique. Nous remercions son directeur, M. Frédéric Crahay, de nous avoir autorisé à le publier.

${ }^{1}$ Entretien électronique du 4 février 2020.

${ }^{2}$ Ibid.
} 


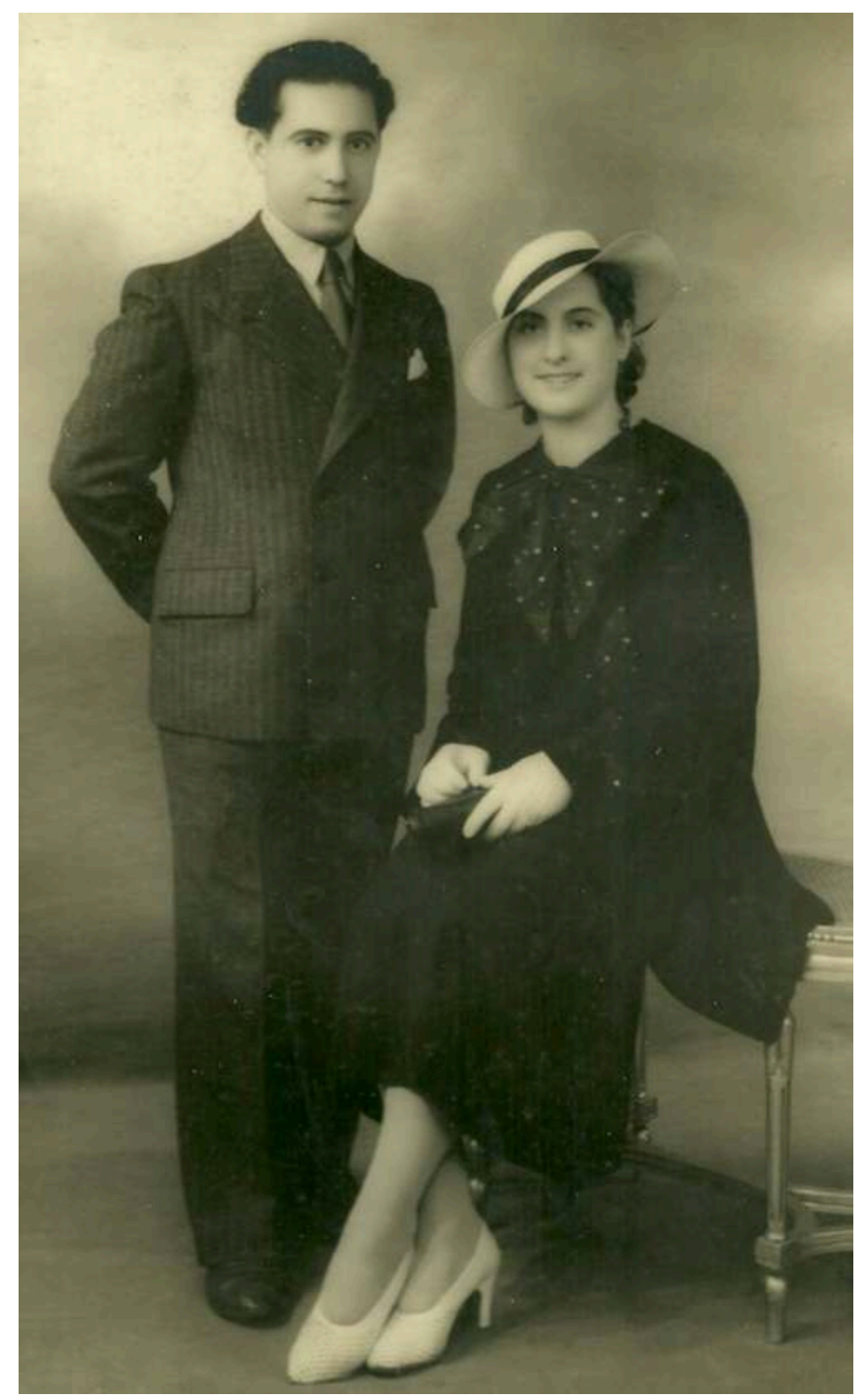

Adolphe et Sosia

Coll. Privée C. Sokolski

Copie digitale Caserne Dossin (KD_00128_000008) 


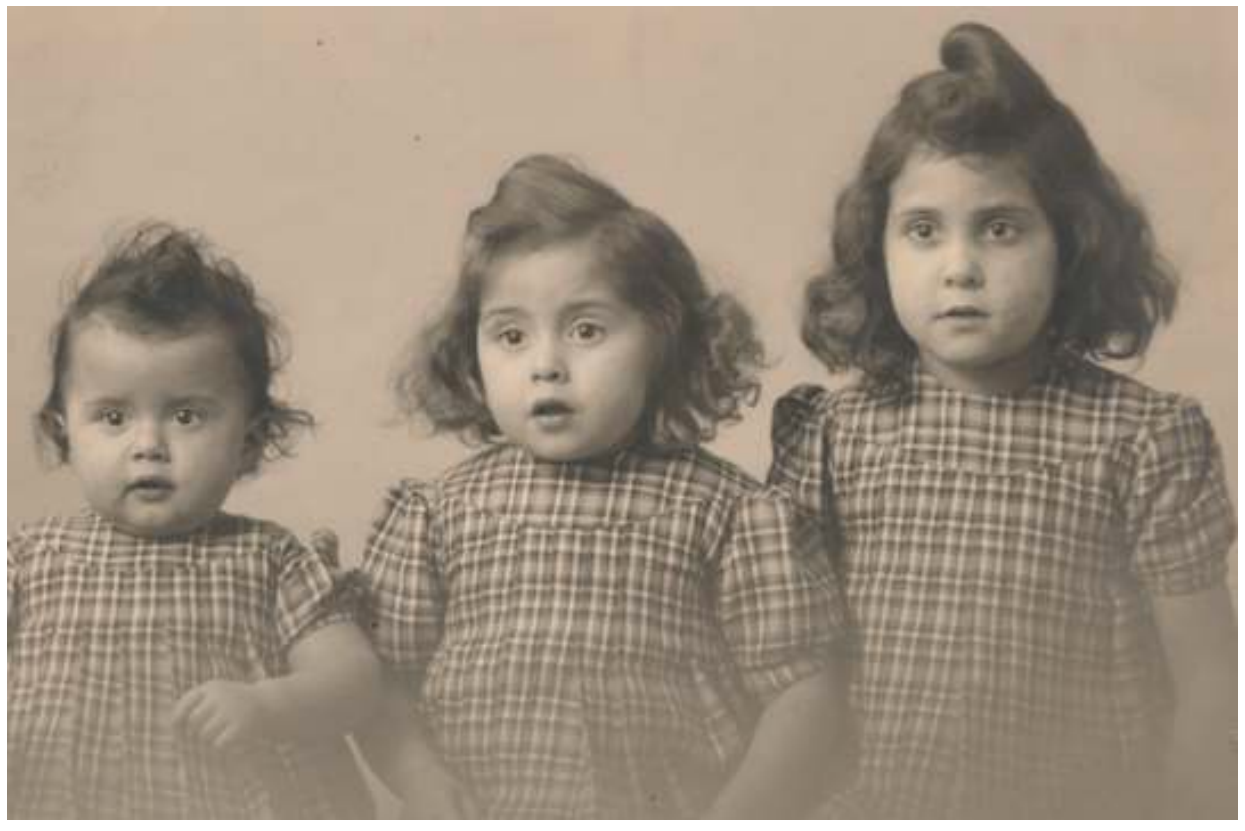

Francine, Arlette et Jacqueline Sokolski (de gauche à droite)

Coll. Privée C. Sokolski

Copie digitale Caserne Dossin (KD_00128_000039)

Les parents manifestent une forte volonté d'intégration à la France, à l'image des nombreux Juifs immigrés dans la région, dans l'entre-deuxguerres, qui croient fermement en la République française et ses valeurs. Le choix des prénoms, très français, des enfants du couple en est une preuve évidente, de même que l'engagement volontaire d'Abraham, en septembre 1939 dans la Légion Étrangère, en France. Celle-ci le renvoie chez lui à Douai car l'usine Cousineau, où il est chef d'atelier, fabrique des uniformes militaires. Enfin, la demande de naturalisation obtenue par Adolphe juste avant-guerre indique, très clairement, le choix de sa nouvelle patrie. Il fut dénaturalisé par le régime de Vichy, comme nombre de Juifs naturalisés après $1927^{3}$.

Georgette Liakhoff se souvient bien de la famille Sokolski :

Nous connaissions bien les Sokolski. C'était une famille agréable avec ses trois petites filles aux cheveux magnifiques. Je jouais souvent avec elles, même si elles étaient beaucoup plus jeunes que moi. Le père, Adolphe, était un petit

\footnotetext{
${ }^{3}$ Témoignage de Claude Sokolski, recueilli par Nathalie Labreigne, La Voix du Nord, 9 septembre 2012 .
} 
homme râblé plein d'allant et d'humour. Il travaillait dans une grosse entreprise de confection. II adorait ses enfants. Le couple formait avec ses filles une famille très unie, agréable, que nous aimions rencontrer. ${ }^{4}$

\section{Les Sokolski, une famille brisée par la Shoah}

Dès octobre 1940, en vertu de l'accord passé entre l'administration militaire allemande, d'un côté, avec les secrétaires généraux des ministères belges et, de l'autre côté, avec le régime de Vichy, les Juifs du Nord-Pas-de-Calais et de Belgique sont tenus de se faire recenser dans les mairies.

Adolphe est arrêté le 22 juin 1941 comme " otage juif bolchevik », puis il est interné six mois au Fort d'Huy en Belgique entre le 21 août 1941 et le 16 mars 1942 et enfin relâché pour une raison inconnue ${ }^{5}$. Adolphe ne goûte pas longtemps à la liberté et à sa vie de famille retrouvées. En juin 1942, Eichman ordonne aux «chargés d'affaires juives » de France, Pays-Bas et Belgique, de procéder à la déportation, à l'été 1942, de 10000 Juifs de Belgique et Nord-Pas-de-Calais ${ }^{6}$.

Pour atteindre cet objectif en Belgique et dans le Nord-Pas-deCalais, deux procédés vont être utilisés par les nazis. Dans un premier temps, en juillet-août 1942, 13000 «Convocations au travail » à l'Est de l'Europe sont distribuées à des Juifs. Ces Juifs « convoqués » sont tenus de se présenter par eux-mêmes à la Caserne Dossin à Malines ${ }^{7}$. Puis dans un deuxième temps, en août-septembre 1942, les nazis lancent quatre grandes rafles sur Anvers (trois) et sur Bruxelles (une). La rafle des Juifs du Nord-Pas-de-Calais du 11 septembre 1942 s'inscrit donc dans cette continuité logique. Pour l'organisation de ce 11 septembre, initié par l'administration militaire allemande, les ordres sont adressés aux différentes Kommandanturen du Nord-Pas-de-Calais. La veille, celles-ci s'adressent à la police locale qui leur délègue des policiers.

La rafle touche tout le bassin minier, comme le Valenciennois où la brigade de gendarmerie de Denain et de Valenciennes se présente vers quatre heures du matin, aux Kreiskommandanturen de ces deux villes. De là, la brigade est répartie en groupes, sous la direction de feldgendarmes,

\footnotetext{
${ }^{4}$ Témoignage de Georgette Liakhoff recueilli par Claude Sokolski, le 30 juillet 2013.

${ }^{5}$ Témoignage de Claude Sokolski, le 17 décembre 2019, à partir du dossier d'Abraham Sokolski conservé par le Service Archives des Victimes (SVG) de la Guerre, Bruxelles.

${ }^{6}$ Maxime Steinberg, Un pays occupé et ses Juifs : Belgique entre France et Pays-Bas, Gerpines-Ottignies, Belgique, éd. Quorum, 1998.

${ }^{7}$ Ibid.
} 
afin de les accompagner aux domiciles des Juifs étrangers. Les rôles sont bien répartis : les gendarmes français assurent la garde et la surveillance des immeubles des Juifs, les nazis procèdent à l'arrestation dans les logements.

Ce jour tragique du 11 septembre 1942, il est donc quatre heures $\mathrm{du}$ matin quand on frappe à la porte des Sokolski. La famille est brutalement tirée du lit par les feldgendarmes aidés de quatre agents de la police française. Les Sokolski, mais aussi trois autres familles juives étrangères, sont ainsi brutalement arrêtées lors de la rafle de Douai, soit un total de quatorze personnes ${ }^{8}$.

Georgette Liakhoff se souvient de la dernière journée avec un sentiment de culpabilité qui persiste 71 ans après :

Ce 11 septembre il faisait très beau. Je m'en rappelle comme si c'était hier. J'ai d'abord vu partir ma tante au petit matin, vers 6 heures. On les emmenait à la gare [de Douai]. Les autres raflés ont suivi. Les gens étaient apparemment calmes [...]. Elles étaient tellement belles et gentilles. On aurait pu les prendre et les sortir sans probleme... Mais aucun parent n'a voulu se séparer de ses enfants... il ne s'agissait que d'un petit voyage... Si on avait su...

Tous les Juifs étrangers raflés sont ensuite rassemblés à la gare de Lille-Fives d'où part un train avec des wagons de troisième classe. Ils parviennent le jour même à la Caserne Dossin à Malines. "L'Aufnahme », « La Réception », dirigée par le tyrannique adjudant SS, Max Boden, est le premier « service » vers lequel les Juifs sont présentés à leur arrivée. Les Juifs y sont inscrits sur une liste pour le prochain convoi par des "Hof Arbeiter », secrétaires juives. La famille Sokolski reçoit les n $148-149-150-151$ et 152.

La famille Sokolski et les Juifs du Nord-Pas-de-Calais arrivés le 11 septembre, quittent la Caserne Dossin dès le 15 septembre 1942. Ce convoi est alors le dixième convoi envoyé à Auschwitz-Birkenau. Il comporte 1047 déportés (537 hommes et 510 femmes), dont 229 enfants de moins de seize ans. La moitié des déportés provient des rafles anversoises des 11 et 12 septembre 1942. L'autre moitié (513 déportés) provient de la rafle des Juifs du Nord-Pas-de-Calais. Le convoi X atteint Auschwitz le 17 septembre ${ }^{10}$.

\footnotetext{
${ }^{8}$ La Voix du Nord, 11 septembre 2012.

${ }^{9}$ Témoignage de Georgette Liakhoff recueilli par Claude Sokolski, le 30 juillet 2013.

${ }^{10}$ Maxime Steinberg et Serge Klarsfeld, Mémorial de la déportation des Juifs de Belgique, Bruxelles, éd. par l’Union des Déportés Juifs de Belgique et Filles et Fils de la Déportation, 1982, description du convoi X, et site numérique de la Caserne Dossin.
} 

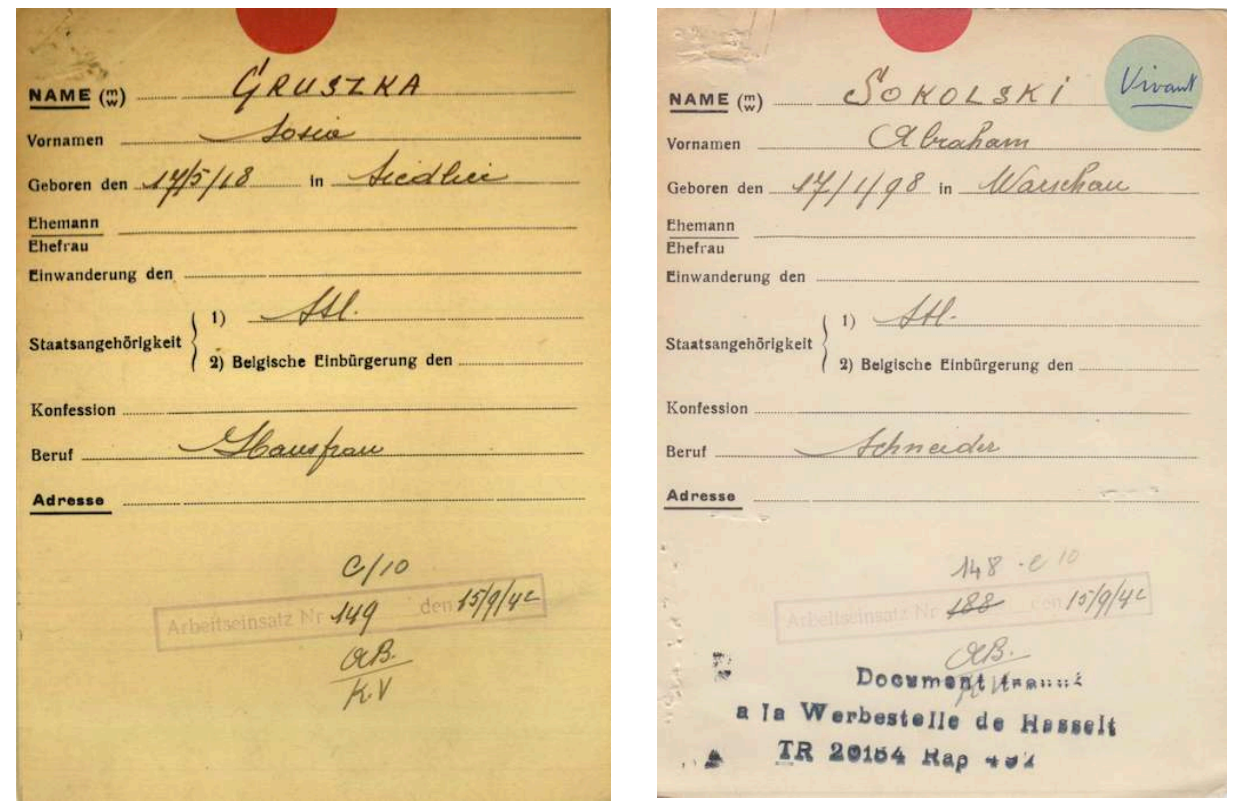

Fiches du SD, à la Caserne Dossin, de Sosia et Adolphe Sokolski (né Abraham) Service Archives des Victimes de la Guerre, Bruxelles

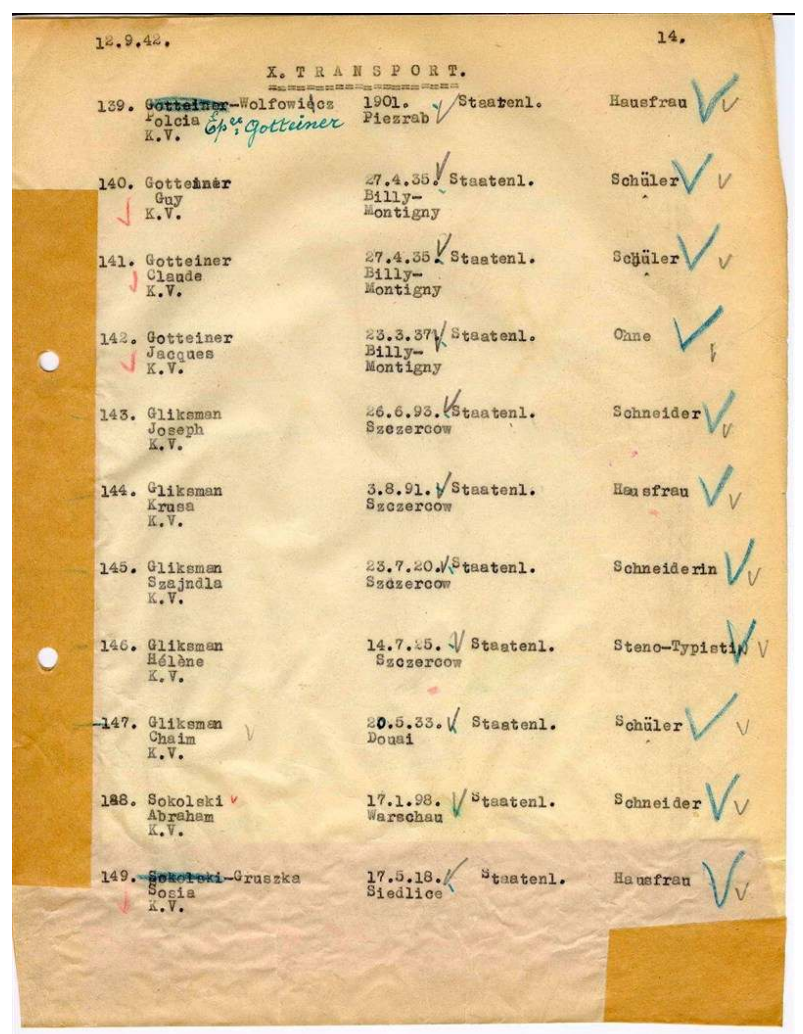




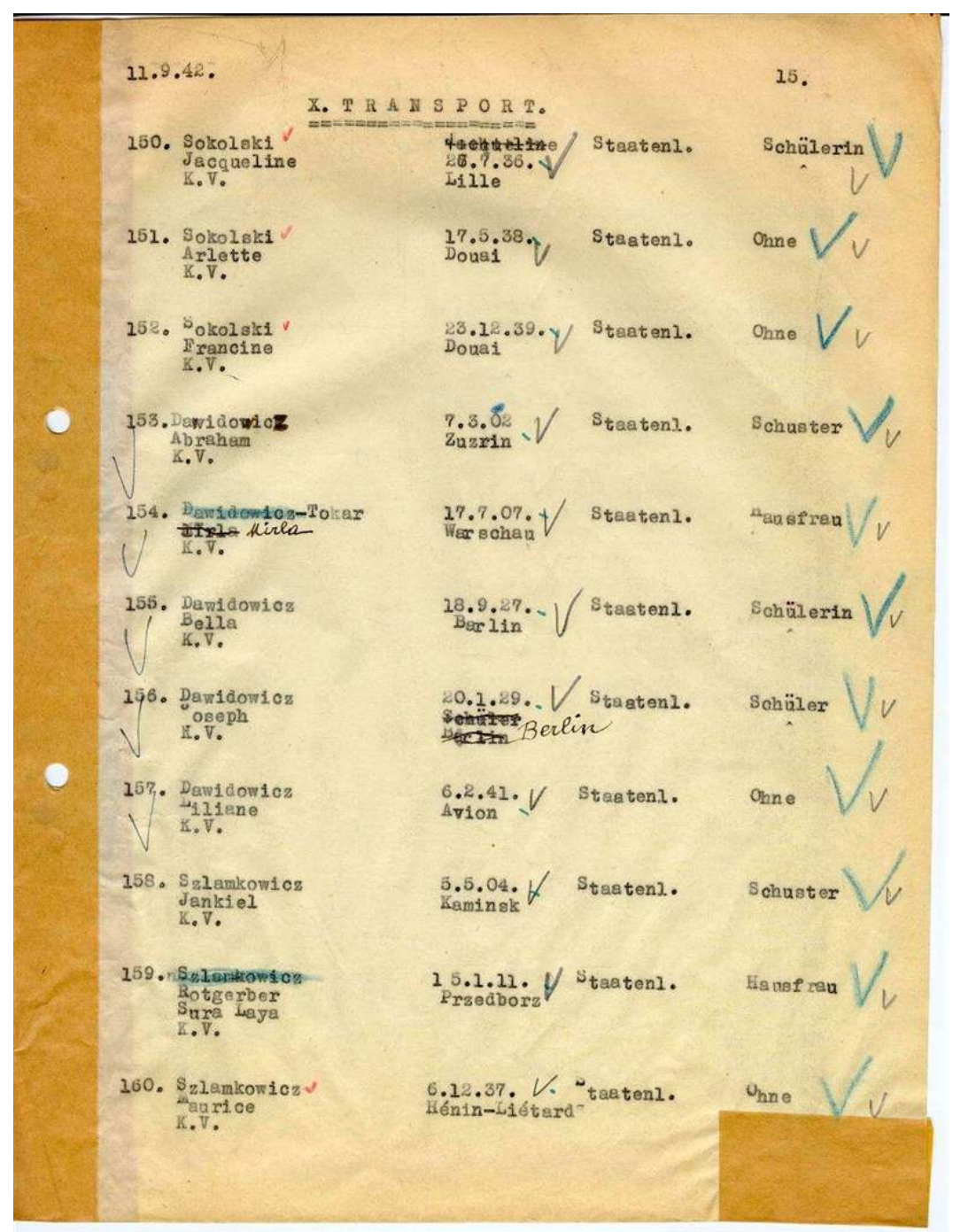

Liste du convoi X avec les noms d’Abraham et Sosia Sokolski n¹48-149 (page précédente, en bas de la liste) et ceux de leurs trois filles $n^{\circ} 150$ à 152 (ci-dessus, en haut de la liste)

Service Archives des Victimes de la Guerre, Bruxelles Copie digitale Caserne Dossin (KD_00013_01_0010_000014)

À l'arrivée, Sosia et ses trois enfants: Arlette, Francine et Jacqueline sont gazées immédiatement. Adolphe passe la sélection pour travailler à Auschwitz (matricule 64095). 
Mon père a passé l'essentiel de sa déportation dans des commandos de travail. Mon père a notamment été envoyé à Varsovie pour participer au "nettoyage » des ruines du ghetto. Il aurait fait partie d'un réseau de solidarité liant les déportés du Nord. À Dachau, il a travaillé dans des usines d'armement nazies telles Buna Monowitz [Auschwitz] ${ }^{11}$.

En effet, le 6 août 1944, il est transféré au camp de concentration de Dachau, puis le 13 au Kommando Mühldorf ${ }^{12}$. Libéré du camp par les Alliés, Adolphe rentre dans le Nord, par train, le 20 mai 1945.

\section{Le retour à la vie et l'intime après la Shoah}

« Mes parents se sont rencontrés à leur retour de camp. Ma mère, Tauba, m'a eu à 50 ans. J'étais son premier enfant $»^{13}$. Adolphe a alors 56 ans. Sa nouvelle femme, Tauba, dite Thérèse, est une Polonaise, née à Varsovie le 22 août 1904. Une émigrée fixée, dans les années vingt dans le département du Nord. Elle y exerce le métier de vendeuse sur les marchés, les carreaux de mines, les routes, avec une charette à bras pour transporter la marchandise. Adolphe travaille à l'usine Cousineau de Lille, plus petite et moins éprouvante que celle de Douai. Il a souffert du dos après la guerre à cause des coups de schlagues reçus en captivité.

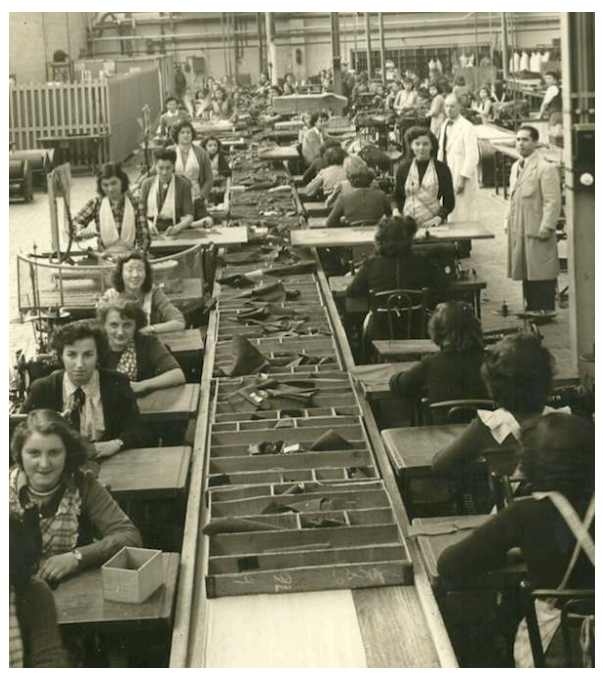

L'usine texile Cousineau à Lille dans les années cinquante

Adolphe Sokolski se trouve à droite debout

Coll. Privée C. Sokolski Copie digitale Caserne Dossin (KD_00128_000007)

\footnotetext{
11 Témoignage de Claude Sokolski, 5 mars 2020. Voir également Claire Zalc, Nicolas Mariot, Face à la persécution. 991 Juifs dans la guerre, Paris, Odile Jacob, 2010, à propos de ce Kommando de travail venu d'Auschwitz et envoyé à Varsovie, p. 214-216.

${ }^{12}$ Dossier d'Abraham Sokolski (fiche du camp), Service Archives des Victimes de la Guerre (SVG), Bruxelles.

${ }^{13}$ Témoignage de Claude Sokolski, 5 mars 2020.
} 
J'ai travaillé chez Cousineau à Douai, rue Giroud de 1936 à 1946. En 1942, j'ai su que la police allemande et française était venue arrêter Monsieur Adolphe et sa famille parce que chez Cousineau, il était connu sous le nom de Monsieur Adolphe. Je me rappelle de lui, d'un homme juste et je ne l'ai jamais entendu crier sur les ouvrières.

Je l'ai revu quand il a recommencé à travailler en 1945. Je vous raconte une anecdote qui m'a touchée. Je pleurais parce que je venais de savoir que ma nièce allait avoir un enfant et qu'elle n'avait pas encore 16 ans. Il est venu me demander pourquoi je pleurais et je lui ai raconté. Il m'a répondu « ce n'est rien d'avoir un enfant à n'importe quel âge, moi, on m'a tué mes 3 enfants ».

En 1942, il avait un beau visage et en 1945, il est revenu avec un visage buriné. ${ }^{14}$

Atteint de la maladie de Parkinson, en 1965, Adolphe, avec Thérèse et Claude, s'installe sur la Côte d'Azur. Sa santé se dégrade rapidement et Adolphe meurt à Menton le 2 avril 1967. Pour Claude, son fils de 13 ans, c'est une page occultée de la vie de son père et de sa mère qui s'entrouve alors. De son vivant, Adolphe a caché à son fils le destin tragique de sa famille. « On m'expliqua, après son enterrement, qu'il avait trois filles Jacqueline, Arlette et Francine, d'une épouse assassinée à Auschwitz $»{ }^{15}$.

Abraham et Taubia veulent tourner la page après la guerre, oublier, aller de l'avant comme l'indiquent ces propos de Thérèse à son fils Claude : «Elle m'a raconté que le jour de ma naissance, mon père avait retiré de son portefeuille la photo de ses trois filles pour la remplacer par ma photo ». Et à propos de son mariage juste après la guerre : «Il était impossible d'imaginer autre chose $\gg{ }^{16}$.

Pour Abraham et Thérèse, en effet, le mariage était le symbole du retour à la vie, à l'intime, au retour à la normalité.

La Shoah a broyé cette famille modeste mais heureuse et unie.

\footnotetext{
${ }^{14}$ Témoignage de Mme Marmousez qui a travaillé comme ouvrière chez Cousineau à Douai, 2015.

15 Témoignage de Claude Sokolski, 3 février 2020.

${ }^{16}$ Ibid.
} 